\title{
3-DIMENSIONAL EAR RECOGNITION BASED ITERATIVE CLOSEST POINT WITH STOCHASTIC CLUSTERING MATCHING
}

\author{
${ }^{1}$ Khamiss Masaoud S. Algabary, ${ }^{2}$ Khairuddin Omar and ${ }^{1}$ Md Jan Nordin \\ ${ }^{1}$ Department of Computer Science, \\ ${ }^{2}$ Center for AI Technology, \\ Faculty Science and Information Technology, University Kebangsaan Malaysia, Bangi, Malaysia
}

Received 2013-08-12; Revised 2013-10-17; Accepted 2013-11-29

\begin{abstract}
Ear recognition is a new technology and future trend for personal identification. However, the false detection rate and matching recognition are very challenging due to the ear complex geometry. The Scope of the study is to introduced a combination of Iterative Closest Point (ICP) and Stochastic Clustering Matching (SCM) algorithm for 3D ears matching based on biometrics field with a good steadiness to reduce the false detection rate. The corresponding ear extracts from the side range image and characterized by $3 \mathrm{D}$ features. The proposed method used matlab simulation and defined the average detection time $35 \mathrm{~ms}$ and an identification similarity is $98.25 \%$ for the collection of different database. The result shows that the proposed combined method outperforms than the existing of ICP or SCM in terms of detection time and accuracy in training.
\end{abstract}

Keywords: Iterative Closest Point (ICP), Stochastic Clustering Matching (SCM), Preprocessing, 3D Ears Matching, Ear Identification

\section{INTRODUCTION}

Illustrations of fake breaches identity card based structures have motivation improved in term of strengthening security via biometrics for auto recognition (Arbab-Zavar and Nixon, 2011; Abaza and Ross, 2010). By means of biometric personalities, the face and ear have acknowledged some important attention due to insensitivity and the simplicity of data collection. Face identification with natural expressions has reached a lot of development with a great value of accuracy (Abaza and Bourlai, 2013; Islam et al., 2008b). However, the variations due to facial expressions, the eye glasses, the use of cosmetics and the incidence of facial hair with beard, they have significantly affected for the performance of face identification. The ear identification, is much smaller in size compared to the face identification, but has a complicated structure (Arbab-Zavar and Nixon, 2007) and a different shape (Zhong et al., 2012) which remains the same shape from 8 to 70 years old as determined by; Zhao et al. (2003). Figure 1 shows the anatomy of the human entire ear. Thus, it is considered to be a suitable alternative method or complement to the face for current human identification (Ossowskia et al., 2013; Hurley et al., 2007; Islam et al., 2008a). A biometric system is a pattern recognition system that establishes the authenticity of a specific physiological or behavioral characteristic possessed by a user. It is regarded as the most secure and accurate authentication tools for verifying human identity (Narendira and Kumar, 2012). With the presence of threats of terrorism and other criminal activity undermining the safety of nations and people, the need for biometric techniques is apparent (Narendira and Kumar, 2012). However, due to the requirement of well-controlled environments or other reasons, most experiments on biometric systems do not live up to their expectations nowadays. As each biometric has its strengths and weakness, no single biometric is expected to effectively meet the requirements of all applications.

Corresponding Author: Khamiss Masaoud S. Algabary, Department of Computer Science, Faculty Science and Information Technology, University Kebangsaan Malaysia, Bangi, Malaysia 


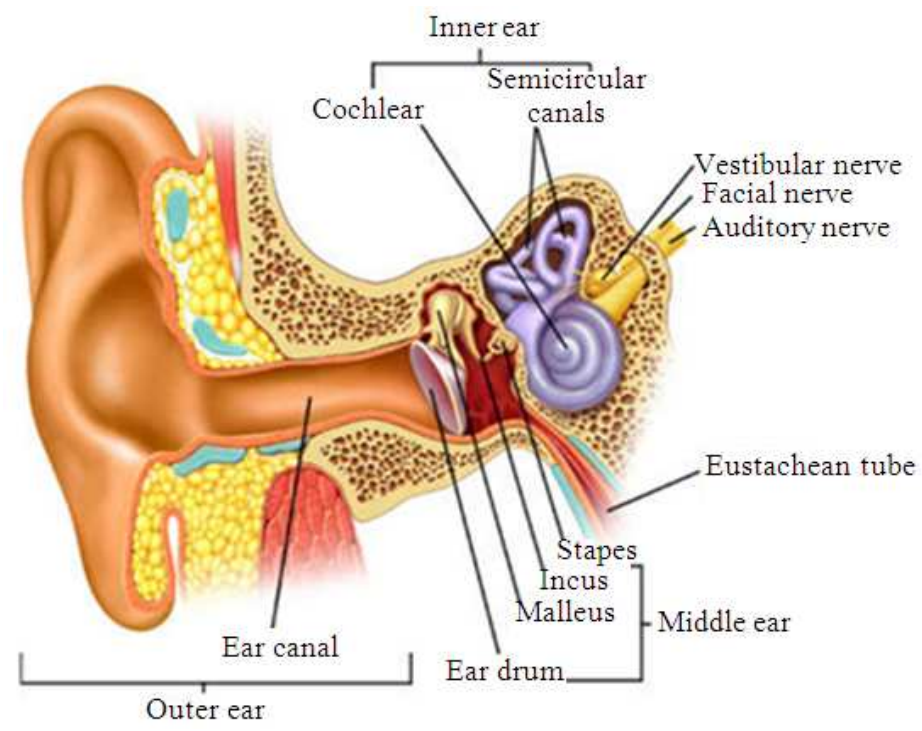

Fig. 1. Anatomy of the human entire ear

The anatomy of the entire human ear is shown in Fig. 1. Nevertheless, in human ear recognition only the outer ear is necessary. The locations of these parts vary for every individual.

\subsection{D Matching Approaches}

The purpose of the new approach for integrated ICP and SCM feature is to be more robust to correspondences parameter to tune the maximum match distance to present in most variants of ICP.

This method proposed to margins the define the expert system based on the combined output of SCM and EBF for segmentation evaluation. The performance of the analyses depends on the evaluated points at various stages of the conversion using Speedup Robust Feature (SURF) analysis. The proposed method designed to allow the selection target to produce automatic feature vector, which is used by a trained the Neural Network to diagnose the classes of calcifications. The system performance was evaluated with a different dataset model to confirm the success rate by obtaining a classification result. A local feature extraction technique (SURF) used to enhance the images to minimize the effect of pose variations and reduced image registration. The SURF feature will be carried out on enhanced images to gain the sets of local features for each enhanced image.

This template has been tailored for output on the custom study size $(21 \times 28.5 \mathrm{~cm})$. The margins are set as follows: top $=25 \mathrm{~mm}$, bottom $=30 \mathrm{~mm}$, right $=20 \mathrm{~mm}$, left $=20 \mathrm{~mm}$, space between column $=75 \mathrm{~mm}$. The paragraphs must be indented. All paragraphs must be left justified and right justified.

\subsection{Iterative Closest Point (ICP)}

The Iterative Closest Point (ICP) algorithm is extensively used for recognizing similarities from 3D models. The ICP algorithm is used to particularly minimize the statistical differences between two sets of data points which commonly referred to as clouds of points. This method is usually used to reconstruct 2-Dimensional (2D) and 3-Dimensional (3D) surfaces from multiple scans. In the field of medical science it is usually used for construction of virtual bone models. The ICP uses a relatively simple algorithm to process $2 \mathrm{D}$ inputs into $2 \mathrm{D}$ or 3D outputs. Note that ICP was originally created and designed for image registration. In other words it measures the dissimilarity between two images. The measure of dissimilarity is equivalent to registration error between two images. Being a method for image registration, it is acknowledged as robust against image rotations of translations. One limitation of ICP as pointed out by Anika et al. (2012) is that it stops the execution of the algorithm too early. They explained that this early stoppage is due to the fact that the algorithm gets stuck in a local minimum. In order to lift this limitation, Anika et al. (2012) suggested that two models be used in its execution. These two models will be coarsely pre-aligned before the 
refinement procedure takes place using ICP. In the study conducted by Ossowskia et al. (2013), they have extracted point clouds for the contour of the ear's outer helix. They then registered these point clouds with the reference model with the use of ICP. In later experiments, Zhong et al. (2012) have used Local Surface Patches (LSP) over point clouds that are present on the ear's outer helix. They then followed the same procedure for registering the LSP with the reference model making use of the ICP. This method greatly improved the performance of the ICP backed ear recognition process: from $93.3-96.63 \%$ for using point clouds and LSP, respectively.

\subsection{Stochastic Clustering Method (SCM)}

It can be deduced from the numerous researches recently discussed that indeed an efficient neural network is necessary to produce an efficient ear recognition system. Moreover, it can also be deduced that different evaluation methods can be employed in order to increase the efficiency of the ear recognition system that is being developed. Note that from the experiments of Mian et al. (2008), it can be seen that image patterns can be converted into different numerical data sets and this data set can be evaluated in groups or in clusters due to the fact that have proven that the 10 distinct ear features can be further expanded into 37 subfeatures. Furthermore, they have shown that the average numerical values of these clustered geometric values are distinct for each individual. Nevertheless, Vidal (2011) have recognized that finding clusters in numerical data such as the geometric values of the distinct features is not a straightforward task. There should be an efficient method for determining relevant data clusters. They have explained that, given a particular set of data, it is essential to know how many natural clusters are hidden in it. This difficulty of finding these natural clusters is increased when there are no additional information about the data set is given. In their research they have shown that the stochastic clustering method can be used to determine the number and the patterns of these natural clusters without other inputs aside from the data set itself.

\subsection{Combine SCM and ICP}

Figure 2 shows the stochastic clustering algorithm and ear biometric based field feature proposed for ear recognition development in digital image processing based neural network techniques.

The study are stated in details of ear identification approach to compute ear features of breast recognition, which are associated with Multilayer Neural Network appearance were used for diagnostic ear recognition estimation. All features, including density; normalized Ear, gray level, homogeneity and gray level sum average were selected by an Artificial Neural Network (ANN) using target classifier for essential diagnosis based on data base evaluation.

\section{MATERIALS AND METHODS}

The study involved general framework for proposed research flow environment to justify the steps as shown in Fig 3. Research design methods are a combination of ideas and concepts developed to be defined through design, observation and analysis the truth of a condition. The prospect of identifying individual persons by the shape of his outer ear was first discovered by the French criminologist Bertillon and refined by the American police officer Iannarelli, who proposed a first ear recognition system based on only seven features (Singh and Verma, 2012).

This research study is building a contribution in three aspects. The first is an applying of ICP based on ear biometrics field feature processing techniques, i.e. ear recognition processing techniques that process the feature representation of an ear considered as an image. The second aspect is the extension of the stochastic clustering algorithm based on specialized feature extraction analysis of differing angulagtions and distance. The third aspect is to combine the two previous features and propose a more complete and accurate ear recognition approach to the problem of SURF abnormality indicative and detection. The role of screening the ear classification with stochastic clustering and ear biometrics based field feature is well established to build an ontology-driven human identification component which enables a better combining of ear properties in order to have a successful identify human in various conditions. This figure provides an intuitive framework for describing method and therefore used to outline the algorithms developed in this research.

Identify a pattern of ear structures and use one of the patterns to identify an individual from the database. This is to underscore the effectiveness of the ear biometric system in identification.

The purpose of the new approach for integrated ICP and SCM feature is shown in Fig. 3 to be more robust to correspondences parameter to tune the maximum match distance to present in most variants of ICP. This method proposed to margins the expert system based on the combined output of SCA and EBF for segmentation evaluation. 


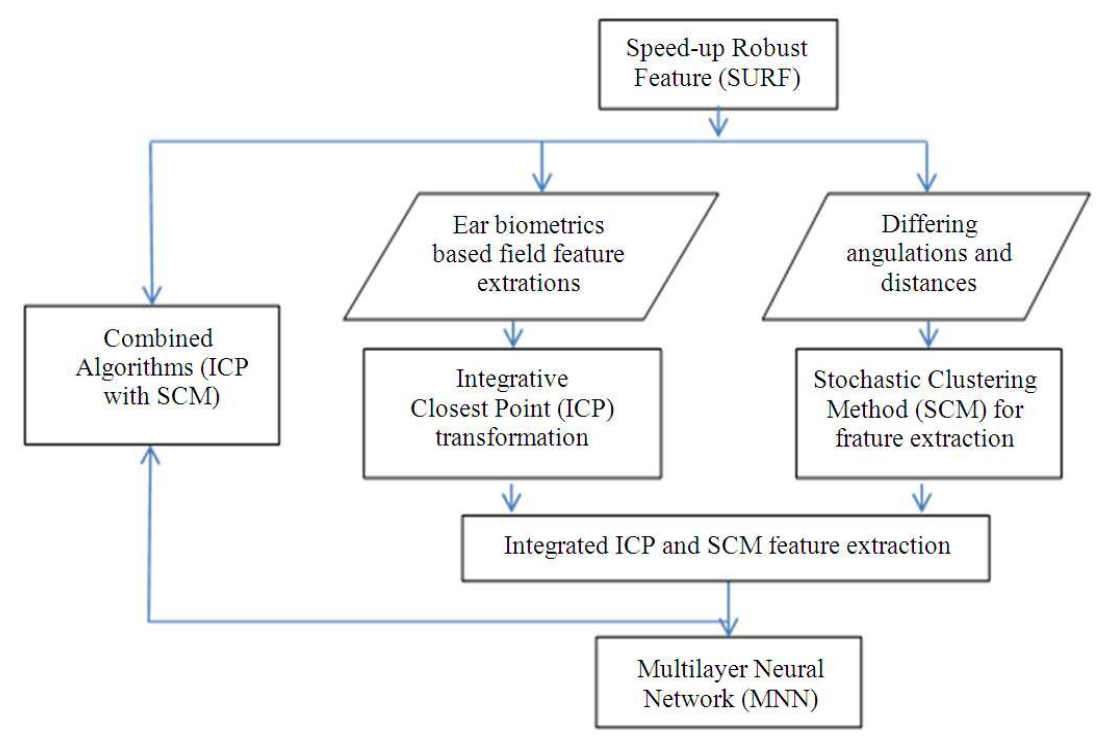

Fig. 2. SCM and ICP Integration

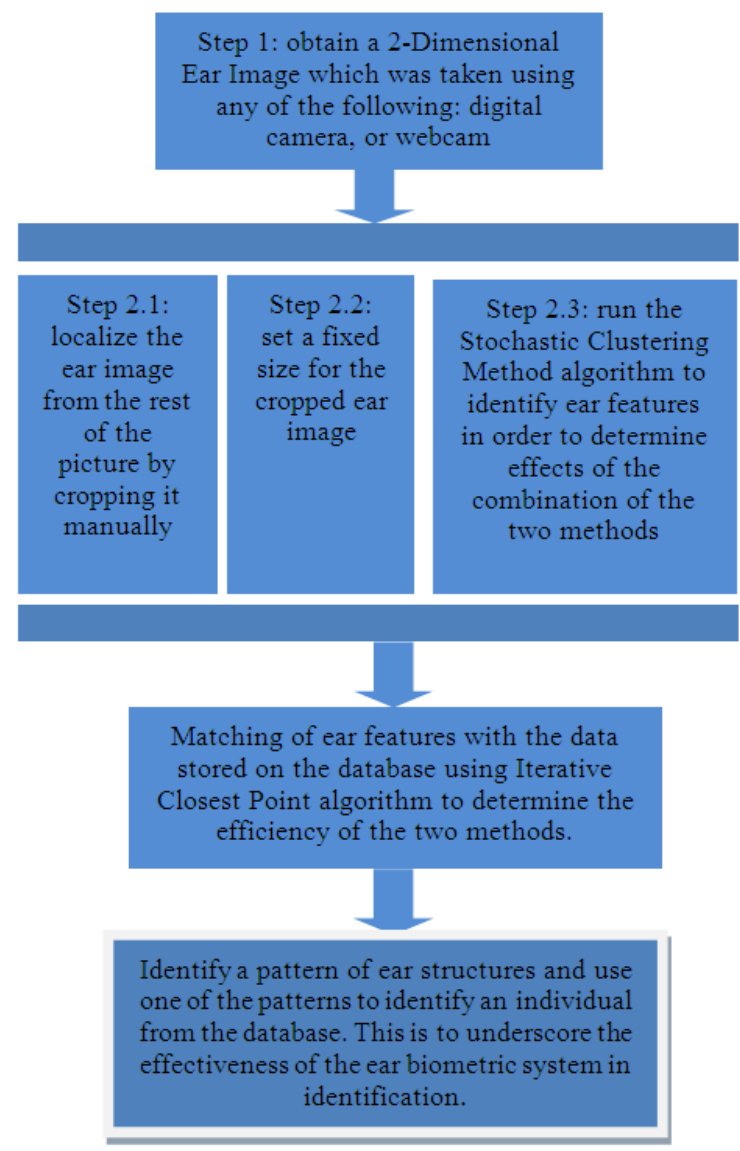

Fig. 3. Proposed research flow environment 


\section{RESULTS}

The performance analyses depend on the evaluated points at various stages of the conversion using Speedup Robust Feature (SURF) analysis. The proposed method designed to allow the selection target to produce automatic feature vector, which is used by a trained the Neural Network to diagnose the classes of calcifications. The system performance will be evaluated with a different dataset model to confirm the success rate by obtaining a classification result.

When using the standard approach done by Anika et al. (2012), before the combination step, the best results are obtained successfully, but more delay is rectified compared with the proposed method as shown in Fig. 4 and 5.

Using the ICP and SCM combinations, very similar results are obtained (consider the standard surface matching based multilayer neural network) as shown in the proposed research flow environment.

These results shown in Fig. 6 confirm the better output discrimination ability of combination method over ICP and SCM and the results reported the obtained prediction axes that the best similarity with the input data with less time are considered.

By analyzing the USTB, IITK and WPUTED database simulations with ICP Fig. 4 and 5 and a combination of ICP with SCM. The following can be concluded.

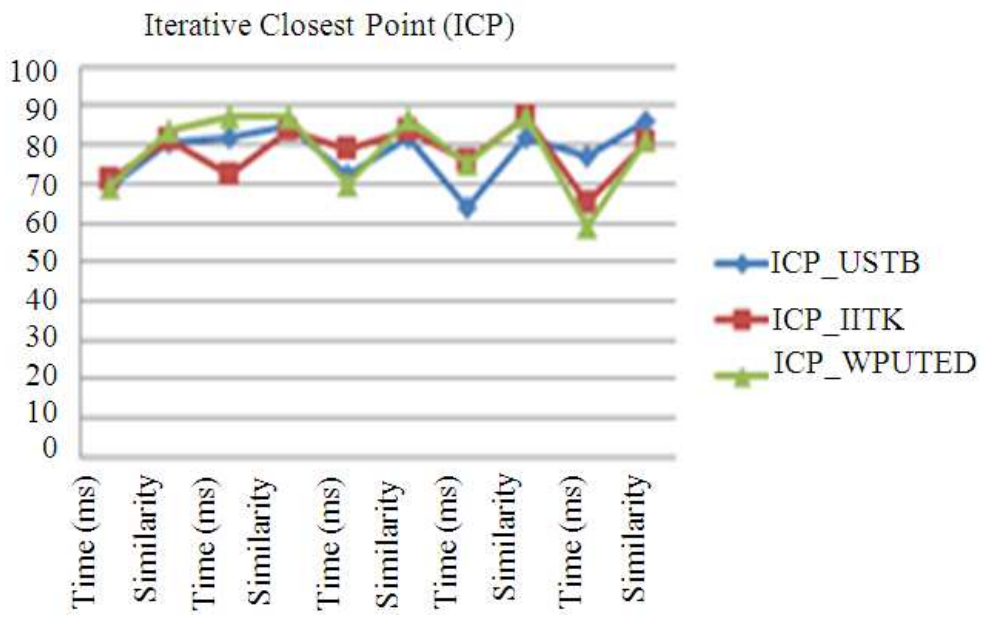

Fig. 4. Ear average detection time using ICP

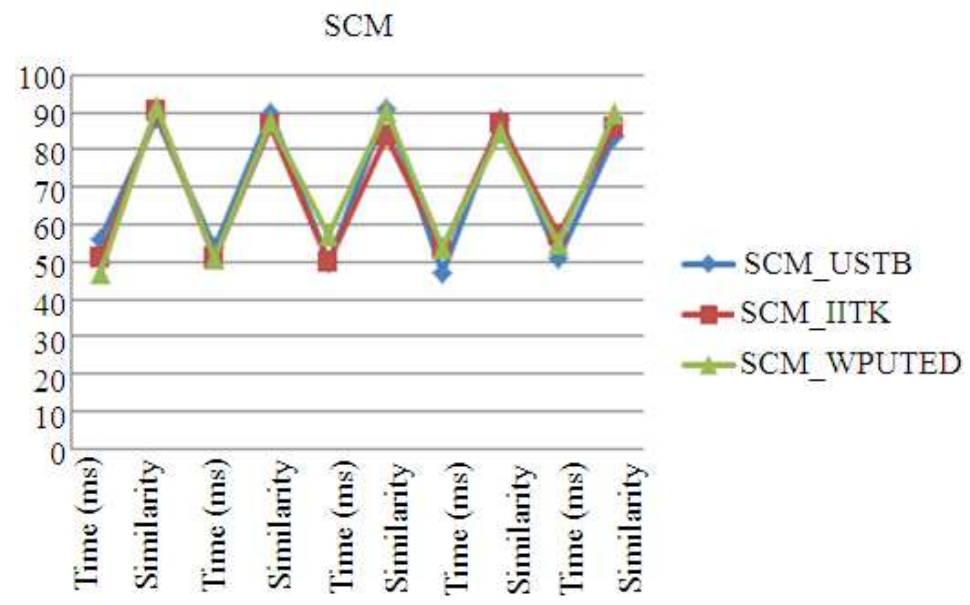

Fig. 5. Ear average detection time using SCM 


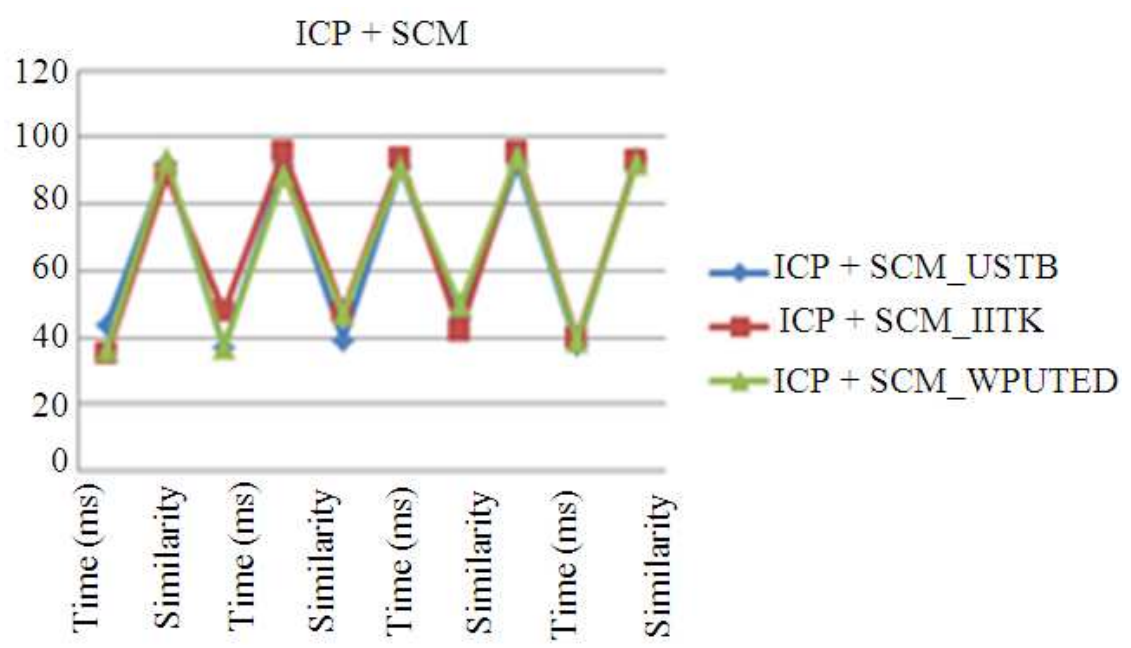

Fig. 6. Ear average detection time using ICP and SCM

\section{DISCUSSION}

A local feature extraction technique (SURF) used to enhance the images to minimize the effect of pose variations and reduced image registration. The SURF feature will be carried out on enhanced images to gain the sets of local features for each enhanced image. The ear image recognition points in the study are fully dependent on Multilayer Neural Networks (MNN). A pre-processing and a post-processing are designed to support ICP algorithm and differing angulations and distance. An anonymous Speedup Robust Feature (SURF) on motivation is delivered after the treatment instruction by the neural network.

As mentioned, ear recognition using combinationmethods work with differences database. In the predifferential case the ear to be subtracted correspond to the ear feature extraction analysis, while in the post-ear identification case they correspond to the multi-layer neural network.

The performed experiments that are conferred in this paper produced good results that indicate the suitability of ears as a reliable biometric to be used in human identification.

\section{CONCLUSION}

In the study, a proposed method is presented in an automated human ear recognition systems, to obtain accurate ear recognition with less time delay using ear biometrics with field feature extractions by Enhancement the ear variations in pose based differing angulations and distances. Furthermore, the method consists of five stages such as preprocessing, feature extraction, Ear classification, Combined algorithms (ICP and SCM) and Ear recognition based MNN ad SURF matching. The recognition rate for ear recognition is measured to be $98.25 \%$. The proposed method stated a new technique that combines Iterative Closest Point (ICP) algorithm with the stochastic clustering algorithm to build ontology-driven human identification multilayer neural network components which enable a better combining of ear properties in order to have a successful identify human in various conditions. The limitation of this research for Ear images have been acquired without any illumination changes. The approach is to be extented to work in varying condition in the future research.

\section{REFERENCES}

Abaza, A. and A. Ross, 2010. Towards understanding the symmetry of human ears: A biometric perspective. Proceedings of the 4th IEEE International Conference on Biometrics Compendium, IEEE Biometrics: Theory Applications and Systems, Sept. 27-29, IEEE Xplore Press, Washington, DC., pp: 1-7. DOI: 10.1109/BTAS.2010.5634535

Abaza, A. and T. Bourlai, 2013. On ear-based human identification in the mid-wave infrared spectrum. Image Vis. Comput. J., 31: 640-648. DOI: 10.1016/j.imavis.2013.06.001

Anika, P., D. Hartung and C. Busch, 2012. Feature extraction from vein images using spatial information and chain codes. Inform. Sec. Tech. Rep., 17: 26-35. DOI: 10.1016/j.istr.2012.02.003 
Arbab-Zavar, B. and M.S. Nixon, 2007. On shapemediated enrolment in ear biometrics. Adv. Visual Comput., 4842: 549-558. DOI: 10.1007/978-3-54076856-2_54

Arbab-Zavar, B. and M.S. Nixon, 2011. On guided model-based analysis for ear biometrics. Comput. Vis. Image Underst., 115: 487-502. DOI: 10.1016/j.cviu.2010.11.014

Hurley, D.J., V. Arbab-Zavar and M.S. Nixon, 2007. The Ear as a Biometric. In: Handbook of Biometrics, Jain, A.K., P. Flynn and A.A. Ross (Eds.), Springer, New York, ISBN-10: 0387710418, pp: 131-150.

Islam, S.M., R. Davies, M.A. Mian and S. Bennamoun, 2008a. A fast and fully automatic ear recognition approach based on 3D local surface features. Proceedings of the 10th International Conference on Advanced Concepts for Intelligent Vision Systems, Oct. 20-24, Springer-Verlag Berlin, France, pp: 081-1092. DOI: 10.1007/9783-540-88458-3_98

Islam, S.M.S., M. Bennamoun and R. Davies, 2008b. Fast and fully automatic ear detection using cascaded adaboost. Proceedings of the IEEE Workshop on Application of Computer Vision, Jan. Jan.7-9, IEEE Xplore Press, Copper Mountain, CO, pp: 1-6. DOI: 10.1109/WACV.2008.4544023

Mian, A., M. Bennamoun and R. Owens, 2008. Keypoint detection and local feature matching for textured $3 \mathrm{~d}$ face recognition. Int. J. Comput. Vis., 79: 1-12. DOI: $10.1007 / \mathrm{s} 11263-007-0085-5$
Narendira, V.K. and B. S. Kumar, 2012. Ear biometrics in human identification system. Int. J. Inform. Technol. Comput. Sci., 2: 41-47.

Ossowskia, A., M. Kusa, P. Brzezinskib, J. Prufferd and J. Piateka et al., 2013. Example of human individual identification from World War II gravesite. Forensic Sci. Int. J., 233: 179-192. DOI: 10.1016/j.forsciint.2013.09.004

Singh, A. and N. Verma, 2012. Ear recognition for automated human identification. Res. J. Eng. Sci., 1: 44-46.

Vidal, R., 2011. A tutorial on subspace clustering. IEEE Signal Process. Mag., 28: 52-68.

Zhao, W., R. Chellappa, A. Rosenfeld, P.J. Phillips, 2003. Face recognition: A literature survey. ACM Comput. Surveys, 35: 399-458. DOI: 10.1145/954339.954342

Zhong, X., Y.S. Wong, W.F. Lu, K.W.C. Foong and A.H.L. Cheng, 2012. A dental matching approach using partial surface features for human identification. Proceedings of the ASME International Design Engineering Technical Conferences and Computers and Information in Engineering Conference, Aug. 12-15, Chicago, Illinois, USA., pp: 357-366. DOI: 10.1115/DETC2012-70890 